\title{
Hepatitis C therapy in 2011: is less more, is more less?
}

Author

Evaldo Stanislau Affonso de Araújo

MD, PhD, Hospital das Clínicas, Medical School, Universidade de São Paulo, SP, Brazil

Correspondence to evaldostanislau@uol.com.br

Conflict of interest: The author declares that in the past 12 months he has received grants for lectures, scientific advisory boards and clinical research from Abbott, Janssen, MSD and Roche.

(ㅇ)2011 Elsevier Editora Ltda. All rights reserved.
The year 2011 will be an extraordinary one. Twenty-two years after the discovery of hepatitis $\mathrm{C}$ virus ( $\mathrm{HCV}$ ) we finally have a therapy that acts directly on the virus. We have entered the era of therapy with direct-acting antiviral agents (DAAs). During therapy evolution, we have always searched for a term that would characterize the current modality. We relinquished the "one size fits all", then the "a la carte" therapy, in which dose customization is essential. We have recently started using response-guided therapy (RGT), in which the duration of therapy is defined according to the moment of HCV non-detection. And now, after the first protease inhibitors (PIs) have been approved, will it be possible to define therapy through a new expression? I believe the discussion and therapeutic decisions will gravitate around the binomial safety-compliance, and then, less is more, or more is less, should be the best definition for this new era.

The addition of PIs generates a higher rate of sustained viral response (SVR) in treatment-naïve patients, relapsers and nonresponders, in cirrhotic patients, African-Americans and those with an unfavorable IL28-B profile. It also minimizes the relevance of other characteristics, usually unfavorable ones, associated with the patient (e.g., high HOMA), and potentially reduces therapy duration. In contrast, this new addition results in adverse effects, complex drug-drug interactions and potential resistance. Finally, there is greater or lesser complexity and high prices. Thus, these are decisions to be made in the near future: who should be treated with triple therapy and how to choose among the available options?

This is not an easy question to answer. First of all, who should be treated? Certainly those infected with genotype 1 . It should be clear to all that new PIs act only on HCV genotype 1 (with evidence of action against genotype 2 and, for boceprevir, also against genotype 3$).{ }^{1}$ The high cost should guide selection criteria, including economic ones. Prioritizing some patients will be a natural choice. In this sense, cirrhotic patients, those with advanced fibrosis or at increased risk of progression (older patients, those with exuberant inflammatory activity), in addition to those previously treated, would be the main candidates. The dilemma to be faced is that patients with milder disease would be the ones to attain the most benefit (with higher rates of SVR), while the afore mentioned priority patients besides having been less studied have shown lower SVR rates (nonetheless overcoming the control group). ${ }^{1-6}$ I do not think the answer will come from a single source, much less from therapeutic, scientific or governmental guidelines. In the context of case-by-case and individual decisions, creating an inflexible policy is a complex task. Moreover, the "price" analysis alone will not suffice. From a financial perspective, a complete pharmacoeconomical analysis seems more reasonable. Therefore, if a "more expensive" therapy brings positive economic impacts in the medium and long term, cost-effectiveness will be proven.

Whether we should treat patients with milder disease is an unanswered question. These patients are in better condition to wait for new therapeutic options that will emerge in the coming years. On the other hand, on an individual basis, to treat patients with milder disease is not inadequate and it is an option that has sound scientific evidence. ${ }^{1-6}$ On the other end of the spectrum, even patients with advanced fibrosis should be analyzed individually. There has been a grouping of "F3" with "F4" (cirrhotic) patients in the analysis of PI pivotal studies. ${ }^{2}$ But do these patients represent the same phenomenon? Is the classification method (liver biopsy or noninvasive methods) appropriate? Are all cirrhotic patients the same, or would cirrhotic patients (by definition) but with good functional reserve be better and more responsive?

The set of factors related to the patient (including the socioeconomic ones), to the virus and to therapy leading to a case-by-case discussion will be crucial in this decision-making 
If deciding who will be treated is a complex task, the choice between the options available to the clinician and the patient - boceprevir or telaprevir - will be another routine decision. In this case, four attributes will be decisive: results (SVR), compliance, genetic barrier to resistance, and safety.

\section{A CLOSER LOOK ON TELAPREVIR AND BOCEPREVIR}

Telaprevir and boceprevir are two different compounds (Figure 1) that belong to the same therapeutic class and share a common site of action, the HCV protease. ${ }^{7}$

Although both are classified among the linear protease inhibitors, when considering the structural differences of these compounds it is natural that their clinical use be distinct in terms of administration, dose, drug-drug interactions and adverse events, despite similar clinical efficacy (SVR equivalent). Table 1 summarizes some of their attributes. ${ }^{8}$

\section{Attribute 1: Attained clinical outcomes (SVR)}

The analysis of phase- 3 studies of telaprevir and boceprevir showed a clear superiority of triple therapy (PegIFN + ribavirin + PI) compared to dual therapy (PegIFN + ribavirin). ${ }^{1-6}$ It is important to emphasize that these are distinct studies, with different populations and therefore their results are not comparable, as there were not head-to-head comparisons of the two agents.

One of the characteristics that emphasizes how different the studied populations were, was the observation that in the control groups the responses were different between the boceprevir and telaprevir studies. ${ }^{1-6}$ It was also noteworthy that the administration of boceprevir was preceded by four weeks of dual therapy with PegIFN and ribavirin (the lead-in phase), while this strategy was used in only one of the telaprevir studies. ${ }^{1,2,4,6}$ Allowing for those caveats, we could emphasize that pooled analysis of overall results showed a difference of $25-28 \%$ higher (63-66\% vs. $38 \%)$ in terms of SVR for treatment-naïve

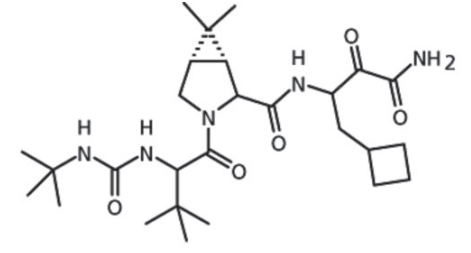

Boceprevir

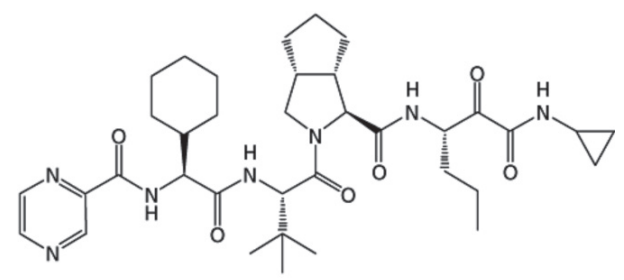

Telaprevir

Figure 1: Chemical structure of boceprevir and telaprevir. ${ }^{5}$

patients who used triple therapy with boceprevir, ${ }^{1,4}$ and $25-31 \%$ (69-75\% vs. $44 \%$ ) for those who used telaprevir (group 12 weeks) compared to standard. ${ }^{1,3.5}$

Regarding those previously treated, a $45 \%$ higher response was obtained in the triple therapy group using boceprevir ( $66 \%$ vs. $21 \%)^{1,2}$ and $47 \%(64 \%$ vs. $17 \%)$ in those who used telaprevir without lead-in, when compared to controls with dual therapy. ${ }^{1,6}$ Among the treatment-naive patients, $44 \%$ to $58 \%$ were eligible for shorter therapy with boceprevir and telaprevir, respectively. ${ }^{1}$ In previously treated individuals, $46 \%$ of those who had used boceprevir received abbreviated therapy. ${ }^{1}$ This information was not available for telaprevir, as the criteria for defining the null response was distinct for boceprevir (patients who, in the lead-in phase, showed less than $1 \log _{10}$ reduction of the viral load). ${ }^{1,2,6}$ Regarding the lead-in phase, it is used with boceprevir based on the results of phase- 3 studies. In phase- 2 boceprevir studies, a direct comparison between using and not using the lead-in showed no significant difference

Table 1. Similarities and differences between first-generation protease inhibitors*

\begin{tabular}{|c|c|c|c|c|c|c|c|}
\hline & Structure & Genotype & Potency & Toxicity & Administration & $\begin{array}{l}\text { Genetic } \\
\text { barrier }\end{array}$ & $\begin{array}{c}\text { Sites of } \\
\text { resistance }\end{array}$ \\
\hline \multirow[t]{4}{*}{ Telaprevir } & Linear & $1 \mathrm{a} / \mathrm{b}$ & High & Rash, anemia & Tid & Low & V36M/A \\
\hline & & & & & & & T54A/S \\
\hline & & & & & & & V55A \\
\hline & & & & & & & $\mathrm{R} 155 \mathrm{~K} / \mathrm{T}$ \\
\hline \multirow[t]{2}{*}{ Boceprevir } & Linear & $1 \mathrm{a} / \mathrm{b}$ & High & Dysgeusia, anemia & Tid & Low & A156S/V/T \\
\hline & & & & & & & V170A \\
\hline
\end{tabular}

*Adapted from Fusco DN, Chung RT. ${ }^{8}$

Tid, three times a day. 
in terms of SVR ( $56 \%$ vs. $54 \%$ in the 28 -week groups, and $75 \%$ vs. $67 \%$ in the 48 -week groups). ${ }^{1}$ There was a trend toward lower breakthrough among patients who used lead-in ( $4 \%$ vs. $9 \%$ ). As in the Realize study, the arm that performed the lead-in with telaprevir showed slight superiority in terms of SVR, particularly among relapsers (88\% vs. $83 \%)$ and null responders (33\% vs. $29 \%)^{1,6}$

It seems that the question of performing the lead-in will be particularly useful in previously treated patients. This initial phase will be important to assess the response to interferon-alpha, to allow pharmacological stability and full effect of ribavirin and to better characterize the type of response of patients whose previous records, for several reasons, prevented a proper classification. In treatment-naïve patients, it may also be useful as a way to measure compliance, evaluate toxicity associated with dual therapy, and even to decide not to include the PIs in the regimen of patients with rapid virological response (RVR) [around $15 \%$ of patients], ${ }^{9}$ whose chance of SVR would be just over $90 \%$ in these circumstances. Therefore, more than a part of boceprevir-associated therapy, the lead-in seems to be an extremely useful tool to categorize patients. On the other hand, not using it is also acceptable for both PIs and clinical practice will certainly allow a better evaluation of future research on the subject.

\section{Attribute 2: Compliance (administration)}

A major difficulty with this first generation of PIs is the pill burden. In case of telaprevir, dosage will be 2 tablets every 8 hours, whereas boceprevir requires intake of 4 pills every 8 hours. Both require 3 daily doses of pills and a number varying between 6 and 12 pills a day. ${ }^{1,10,11}$ The telaprevir therapy lasts for 12 weeks, while boceprevir therapy duration will vary from 24 to 32 weeks, unless early withdrawal interrupts therapy. ${ }^{2,411}$ Consider that it might be necessary to associate the use of pegylated interferon to both drugs, ribavirin and other drugs to control any adverse symptoms and/or concomitant diseases.

The total treatment time may be $24,28,36$ or 48 weeks, considering the lead-in and the possibility of RGT in treatment-naïve patients (24-28 weeks for telaprevir and boceprevir, respectively) or previously treated individuals (36 weeks, boceprevir) or full therapy (48 weeks for both). ${ }^{1-6}$ The number of pills, dose interval and therapy duration are therefore critical. But it is also necessary to consider the matter of toxicity and drug-drug interactions, which will be discussed later, and dietary restrictions. Boceprevir should be taken with a light meal. ${ }^{10}$

Telaprevir should be taken 30 minutes after a caloric (520 calories) and high-fat meal ( $21 \mathrm{~g})$, or there will be significant loss of absorption (up to $237 \%$ lower if administered in the fasting state). ${ }^{11}$ Therefore, patients with comorbidities such as diabetes, dyslipidemia, cerebrovascular or coronary disease will be even more complex to manage. That is, in my opinion, the first critical aspect to be discussed with the patient is: how many tablets is he/she willing to swallow a day and for how long (more or less?). The second aspect will be discussed in the Safety item.

\section{Attribute 3: Genetic barrier}

The first-generation PIs share the same problem - a low genetic barrier. Monotherapy rapidly induces the selection of resistant variants.

Therefore they must be necessarily used in conjunction with pegylated interferon and ribavirin (one must be careful to avoid the use of an unintended monotherapy, for instance, in patients with poor compliance to interferon and ribavirin or in those who have to interrupt dual therapy). Furthermore, the subtype 1a has a lower genetic barrier than subtype $1 \mathrm{~b}$, which requires two amino acid substitutions in the codons of viral protease, compared to only one for subtype 1a. In order to prevent expanding the selection of resistant variants and increasing viral fitness, one must be strict when following the rules established for each PI interruption. ${ }^{1}$ In the case of treatment-naïve patients using boceprevir, detection of $\geq 100 \mathrm{IU} / \mathrm{mL}$ at weeks 12 or 24 , or $\geq 1000 \mathrm{IU} / \mathrm{mL}$ at week 4 or 12 , or detected at week 24 when using telaprevir should result in therapy withdrawal. With boceprevir for patients previously treated, detection of $>100 \mathrm{IU} / \mathrm{mL}$ at week 12 , and in the case of telaprevir, $>1000 \mathrm{IU} / \mathrm{mL}$ at week 4 or 12 , should result in therapy withdrawal. ${ }^{1}$ For both, as previously discussed, therapy interruption should also occur if pegylated interferon or ribavirin have to be removed from the regimen. The pattern of mutation that confers resistance to both IPs is similar (Table 1). Therefore, there is cross-resistance and the rescue of one agent by the other is not recommended. New data regarding the persistence, variability and the behavior of these resistant variables have been reported and it is likely that new recommendations related to the management of resistance will be made soon.

\section{Attribute 4: Safety}

It is known that clinical trials are carried out with highly selected populations and do not necessarily represent "real life" patients. The interpretation of this strategy may be to protect the drug compound being developed from a worstcase scenario, or simply to minimize interference factors that might overshadow the real purpose of the trial, and demonstrate the effectiveness of product A or B. Considering these facts, what is observed in registry studies is the efficacy of the drug. Its effectiveness will be the result of the attributes that were analyzed (efficacy, compliance, genetic barrier and safety) applied to real-life patients. Thus, one shall not be surprised to find results that are not identical to those described in registry studies. In the specific case of PIs, the question of compliance will be relevant, but the capacity to maintain patients on therapy will be 
decisive! In general, in phase-3 studies, adverse events leading to therapy discontinuation occurred in $12-16 \%$ of patients with boceprevir (versus $16 \%$ in controls) and $16.5 \%$ in those using telaprevir (versus $4.1 \%$ in controls). ${ }^{1-6}$ Therefore, safety will be the main factor to keep patient on therapy. The item safety should be divided into two topics: drug-drug interactions and adverse effects.

Telaprevir is a substrate and an inhibitor of cytochrome P3A (CYP3A) and P-glycoprotein (P-gp)., ${ }^{1,12}$ It inhibits both CYP3A and P-gp. The use of drugs that depend on the metabolization by this CYP3A pathway is contraindicated due to the potential increase of its plasma concentration, leading to significant toxicity. One example of this mechanism is simvastatin. ${ }^{1,12,13}$ Immunosuppressive agents such as cyclosporine and tacrolimus administered concurrently with telaprevir also showed extremely high levels, to the point of severe toxicity risk. ${ }^{14}$ Other interactions may occur with compounds that interact with these metabolic and transportation pathways. Boceprevir is a substrate of $\mathrm{P}$-gp, which is partially metabolized by CYP3A, also inhibited by it. ${ }^{1,13,15}$ Therefore, it also undergoes the complex interactions with drugs that depend on the CYP3A pathway, such as simvastatin. Another drug that is contraindicated with both PIs is rifampicin, for it induces CYP3A and reduces the concentrations of both PIs. ${ }^{12,13,15}$ Furthermore, benzodiazepines such as midazolam are formally contraindicated for concomitant use with PIs. ${ }^{1,2,13,15}$ The use of concomitant medications has been the subject of specific retrospective analysis of phase-3 studies soon to be presented, with new information on safety. However, it is advisable that prescription of PIs be made after consulting specific sites with updates on the safe use of drugs together with the PIs. One recommended source is www.hep-druginteractions.org. The complexity of potential interactions is a relevant factor when making the decision to delay therapy. Both in elderly patients and those who will grow old until they are treated, the potential of diagnosis of new comorbidities that require specific therapy must be balanced against initiating therapy against HCV in a stage of life where such risk of interaction is absent.

As for safety itself, it is undeniable that we are currently living a time of transition. We left the highly controlled clinical trials for the therapeutic use of these new drug compounds in real life. Out here, there is no selection, there is no easy care. This is about the physician, the patient and the social environment, including available assistance resources. Clearly, we have entered a new era. New adverse effects emerged from the management of hepatitis $C$ therapy. In addition to the known hematologic toxicity, especially anemia, we have been introduced to dermatological and systemic events with lethal potential, and less severe, but equally important events, such as anorectal symptoms and dysgeusia. It is also true that diagnostic and therapy management rules have been determined to handle skin events and the publication of scientific articles addressing adverse skin events is growing. ${ }^{16,17}$ Finally, the dermatologist will be a new member of the multidisciplinary team and the use of erythropoietin should be more frequent.

In phase- 3 studies, anemia was observed with both PIs. Hemoglobin $<10 \mathrm{~g} / \mathrm{dL}$ occurred in $49 \%$ of patients receiving boceprevir ( $29 \%$ in controls) and $9 \%$ had hemoglobin $<8.5 \mathrm{~g} / \mathrm{dL}$. Erythropoietin was used in $45 \%$ of patients $(24 \%$ in controls). However, discontinuation due to anemia was low, $2 \%$, as compared to $1 \%$ among the controls. Likewise, therapy withdrawal due to serious adverse events was comparable between control and boceprevir ( $11 \%$ vs. $8 \%)^{1,2,4}$ Patients who used telaprevir with hemoglobin $<10 \mathrm{~g} / \mathrm{dL}$ were $36 \%$ (14\% in controls) and 9\% had hemoglobin $<8.5 \mathrm{~g} / \mathrm{dL}$. The use of erythropoietin was prohibited in telaprevir studies. Thus, 5-6\% of patients with anemia using telaprevir had to interrupt the therapy. ${ }^{1,3,5,6}$

In France, according to data presented by Hézode ${ }^{18}$ at the National French Liver Meeting in 2011, of 109 patients taking boceprevir, $21 \%$ had hemoglobin levels between 8 and $10 \mathrm{~g} / \mathrm{dL}$ and $6 \%<8 \mathrm{~g} / \mathrm{dL}$. Erythropoietin was used in $41 \%$ of them and blood transfusion in $4 \%$. In relation to telaprevir, of 107 patients, $24 \%$ had hemoglobin levels between $10 \mathrm{~g} / \mathrm{dL}$ and $8 \mathrm{~g} / \mathrm{dL}$ and $13 \%<8 \mathrm{~g} / \mathrm{dL}$. Erythropoietin was used in $45 \%$ of the cases and $17 \%$ required blood transfusions. This is a very different scenario from that in clinical studies. Anemia seems to be a common event with telaprevir and boceprevir therapy, with similar management and magnitude, but with different severity, which may eventually reflect some particular clinical feature of the assessed population.

We must also consider the fact that severe anemia $(\mathrm{Hb}<8.5 \mathrm{~g} / \mathrm{dL})$ occurred in less than $10 \%$ of patients. Most patients with anemia are in the range of usual therapeutic management for the clinicians who already dealt with interferon alpha-based therapy. In addition, ribavirin dose reduction is an effective, alternative measure to erythropoietin use and does not interfere with therapy effectiveness.

Dysgeusia was reported in 35\% of patients who received boceprevir (16\% in controls) and $10 \%$ of patients who received telaprevir ( $3 \%$ in controls). Anorectal events were reported only in patients who received telaprevir (26.2\% vs. $5 \%$ in controls). Initially these events were reported as "hemorrhoids" and later referred to as anal itching, anorectal discomfort and hemorrhoids. The onset occurs in the first two weeks of therapy and its mechanism is unknown. Proctologic examination is usually normal and not associated with skin alterations or the presence of pruritus. There is no standard therapy and progressive improvement occurs after telaprevir withdrawal. ${ }^{1-6}$

Finally, let's address dermatologic events of phase-3 studies. ${ }^{1-6}$ Skin rash was observed in $17 \%$ of patients using boceprevir. However, when compared to controls, in this group, the presence of rash was $19 \%$. Therefore, it is not reasonable to believe that this effect is explained only 
by the boceprevir, as we know that ribavirin is also associated with adverse skin events. On the other hand, $56 \%$ of patients using telaprevir had rash compared with 32\% of controls. In most cases it was mild to moderate, but $4 \%$ of them had more than $50 \%$ of body surface affected and in $7 \%$ of cases, it led to therapy withdrawal. Pruritus occurred in $50 \%$ of patients taking telaprevir whereas Stevens-Johnson syndrome or drug-related eruption with systemic symptoms (DRESS) was reported in approximately $1 \%$ of patients using telaprevir. That is a severe, potentially lethal manifestation and one that requires permanent telaprevir withdrawal. In this case, only as speculation, it is possible to consider the use of boceprevir as PI rescue in order to maintain the potential to treat hepatitis $C$ when the condition is under control. The data reported so far are quite relevant. Hézode ${ }^{18}$ identified $7 \%$ of severe rash among the 107 patients followed in France. Therefore, we must be alert and prepared to diagnose and manage these events, highlighting what the use of these drugs could represent in a country with tropical characteristics (hot, humid and sunny) such as Brazil.

Concerning future discussions with our patients, we must yet again openly discuss what risks he or she is willing to take - more or less? The options to be offered are a longer treatment with more pills, but with fewer adverse events (anemia, dysgeusia) - more is less - or a shorter and simpler treatment, but with a higher risk of adverse and severe events (anemia, dysgeusia, anal discomfort, allergic and dermatologic events) - less is more. The risk associated with PIs is greater than that with dual therapy. This must be made clear to the doctor and patient. Thus, the balance between compliance and safety will not be decided only by the physician, but also by the adequately informed patients and their families.

\section{CONCLUSIONS}

We have finally got what we were demanding: better hepatitis $\mathrm{C}$ therapy! We, forever dissatisfied individuals, must recognize that therapy is still far from what is desired. It is not $100 \%$ effective, it is less effective in cirrhotics and nonresponders, it is not pan-genotypic, it can select resistant variants, it is complex and, above all, it brings new and potentially lethal adverse events and interactions.

On the other hand, it is superior, regarding all aspects and comparisons, to dual therapy with pegylated interferon and ribavirin. It also allows reducing therapy time from the current 48 to 72 weeks to 24 to 28 weeks in most patients, and up to 36 weeks in previously treated ones.

This therapy also has a better cost-effectiveness and may be used, as we soon shall see, in patients coinfected with HIV. The American Association for the Study of Liver Diseases (AASLD) has recommended triple therapy as the standard therapy to those infected by genotype $1 .{ }^{1}$ Thus, before we fear it, we must get ready for it. And above all, we must decide: whom to treat, how to treat? It is my opinion that candidates for therapy will increase in numbers. If we consider these drugs to be cost-effective, all those infected with genotype 1 that require therapy should be treated. If we are more conservative, all those previously treated without success should be treated. If we are even more conservative, those with advanced fibrosis, above F3, should be treated. The only thing we cannot afford to do is not to decide. The prospect that new drug compounds and new DAA classes are to be made available is real. The problem is that it will not ensue immediately. Conservatively, perhaps we will have new therapeutic agents available in the next three to five years. This is a time period that many patients, unfortunately, cannot afford to wait.

\section{REFERENCES}

1. Ghany MC, Nelson DR, Strader DB, et al. An update on treatment of genotype 1 chronic hepatitis $\mathrm{C}$ virus infection: 2011 practice guideline by the American Association for the Study of Liver Diseases. Hepatology. 2011;54:1433-44.

2. Bacon BR, Gordon SC, Lawitz E, et al. for HCV RESPOND-2 Investigators. Boceprevir for previously treated chronic HCV genotype 1 infection. N Engl J Med. 2011;364:1207-17.

3. Jacobson IM, McHutchison JG, Dusheiko G, et al. for ADVANCE Study Team. Telaprevir for previously untreated chronic hepatitis C virus infection. N Engl J Med. 2011;364:2405-16.

4. Poordad F, McCone J Jr, Bacon BR, et al. for SPRINT-2 Investigators. Boceprevir for untreated chronic HCV genotype 1 infection. N Engl J Med. 2011;364:1195-206.

5. Sherman KE, Flamm SL, Afdhal NH, et al. Response-guided telaprevir combination treatment for hepatitis $\mathrm{C}$ virus infection. N Engl J Med. 2011;365(11):1014-24.

6. Zeuzem S, Andreone P, Pol S, et al. for REALIZE Study Team. Telaprevir for retreatment of HCV infection. N Engl J Med. 2011;364:2417-28

7. Cheng K-C, Gupta S, Wang H, et al. Current drug discovery strategies for treatment of hepatitis $\mathrm{C}$ virus infection. J Pharm Pharmacol. 2011;63(7):883-92.

8. Fusco DN, Chung RT. New protease inhibitors for HCV Help is on the way. J. Hepatology. 2011;54:1087-9.

9. Desai AP, Reau N. Naives, nonresponders, relapsers: who is there left to treat? Clin Liver Dis. 2011;15(3):483-95.

10. Boceprevir drug label - FDA.

11. Telaprevir drug label - FDA.

12. No authors listed. Telaprevir PK fact sheet 2011. University of Liverpool (Available at: www.hep-druginteractions.org)

13. No authors listed. The Medical Letter 2011;53(1369):57-59.

14. Garg V, van Heeswijk R, Lee JE, et al. Effect of telaprevir on the pharmacokinetics of cyclosporine and tacrolimus. Hepatology. 2011;54;20-7.

15. No authors listed. Boceprevir PK fact sheet 2011. University of Liverpool (Available at: www.hep-druginteractions.org)

16. Cacoub $\mathrm{P}$, Bourlière $\mathrm{M}$, Lübbe J, et al. Dermatological side effects of hepatitis $\mathrm{C}$ and its treatment: Patient management in the era of direct-acting antivirals. J Hepatol. 2011;Ago 30. [Epub ahead of print]

17. Mountataudié H, Passeron T, Cardot-Leccia N, et al. Drug rash with eosinophilia and systemic symptoms due telaprevir. Dermatology. 2010;221:303-5.

18. Hézode C. National French Liver Meeting, 2011 (oral presentation). 